\title{
Curcumin ameliorates experimental autoimmune acute myocarditis in rats as evidenced by decrease in thioredoxin immunoreactivity
}

\author{
A.M. Ahmed ${ }^{1}$, A.F. El Fouhil ${ }^{1}$, R.A. Mohamed ${ }^{1,2}$, M. Atteya ${ }^{2,3}$, N.A. Abdel-Baky, ${ }^{4}$, \\ A.H. AlRoalle ${ }^{3}$, A.M. Aldahmash ${ }^{3}$ \\ ${ }^{1}$ Department of Anatomy, College of Medicine, King Saud University, Riyadh, Saudi Arabia \\ ${ }^{2}$ Department of Histology, Faculty of Medicine, Cairo University, Cairo, Egypt \\ ${ }^{3}$ Stem Cell Unit, Department of Anatomy, College of Medicine, King Saud University, Riyadh, Saudi Arabia \\ ${ }^{4}$ Department of Pharmacology, College of Pharmacy, King Saud University, Riyadh, Saudi Arabia \\ ${ }^{5}$ Department of Pharmacology, College of Pharmacy, Al-Azhar University, Cairo, Egypt
}

[Received 25 November 2014; Accepted 7 January 2015]

This study was performed to investigate the effect of curcumin on cardiac myosin-induced autoimmune myocarditis in rats and the change in thioredoxin (TRX) immunoreactivity in cardiomyocytes following curcumin treatment. Twenty-four six-week-old male Wistar rats were randomly allocated into 4 groups of 6 rats each. Group I received neither curcumin nor myosin. Group II received an oral solution of $1 \mathrm{~g} / \mathrm{kg} /$ day of curcumin daily, from day 1 to day 21. To induce myocarditis, animals of both group III and group IV were injected by $1 \mathrm{mg}$ of porcine cardiac myosin on days 1 and 8 . In addition, animals of group IV received an oral solution of $1 \mathrm{~g} / \mathrm{kg} /$ day of curcumin daily, from day 1 to day 21. Serum levels of creatine phosphokinase, troponin-T, tumour necrosis factor-alpha and interleukin- 6 were estimated. Hearts were processed for histopathological and immunohistochemical studies. Serum biomarkers levels were significantly increased in myocarditis group as compared to other groups. The intake of curcumin significantly reduced the deviation in these markers. Sections of the wall of the heart from myocarditis group were characterised by inflammatory cell infiltration. Most of cardiomyocytes showed pyknotic nuclei and increased sarcoplasmic eosinophilia with strong immunoreactivity for TRX. Sections from myocarditis-curcumin group showed normal architecture with moderate immunoreactivity for TRX. The present study demonstrated that curcumin ameliorates acute myocarditis in rats and encouraged the estimation of serum level of TRX as a relevant indicator for the evaluation of the progress of acute myocarditis. (Folia Morphol 2015; 74, 3: 318-324)

Key words: curcumin, acute myocarditis, thioredoxin, immunohistochemistry

\section{INTRODUCTION}

Heart disease is one of the major health problems of advanced as well as developing countries in the world. In human, acute myocarditis is a potentially lethal disease, and frequently precedes the development of dilated cardiomyopathy [2].
Myocarditis is defined as myocardial inflammation accompanied with oedema, cellular infiltration, apoptosis, and necrosis of cardiomyocytes [13]. In acute myocarditis, an imbalance of the reactive oxygen species (ROS) and/or an inadequate cellular antioxidant defence mechanism may play a key role in myocardial

Address for correspondence: Prof. A.F.I. El Fouhil, Department of Anatomy and Embryology, College of Medicine, King Saud University, PO Box 2925 (28), Riyadh 11461, Saudi Arabia, tel: +966(11)4671314, mobile: +966501562983, fax: +966(11)4671300,

e-mails: ahmedfathala@gmail.com, aelfouhil@ksu.edu.sa 
injury [20]. Increased ROS are able to induce severe cardiovascular dysfunction by their direct attack on intercellular biomolecules such as contractile molecules or ion channels. Also, the imbalance of intracellular oxido-reductive state (redox) may lead to the activation of stress sensitive signalling pathways, increasing apoptosis and potentially contributing to the development of heart failure [18].

Cells have developed elaborate defence systems against oxidative stresses. Among those, a pivotal role of the thiol-mediated redox systems has been recognised. Thioredoxin (TRX) is a redox regulatory protein that protects the cells from various stresses by reducing activity of oxidised thiol groups on proteins. It was reported that TRX is up-regulated by acute inflammatory stimuli and may play an important protective role in the pathogenesis and development of myocarditis [14]. There are two major TRX: thioredoxin 1 (TRX1), a cytosolic and nuclear form, and thioredoxin 2 (TRX2), a mitochondrial form [16]. TRX1 binds with a variety of cellular proteins and inhibits apoptosis [1].

Cardiac myosin-induced myocarditis is a model of experimental autoimmune myocarditis (EAM) in rats to investigate autoimmunological mechanisms in inflammatory heart diseases. During the development of the disease, myosin first elicits a focal inflammation mediated by neutrophil and macrophage infiltration, which then triggers the activation of mononuclear effector cells and initiates the EAM [3]. This model of EAM is histologically similar to human myocarditis in acute phase, with the recruitment of macrophages, lymphocytes and neutrophils accompanying myocardial fibrosis $[8,20]$.

Curcumin, a yellow pigment from Curcuma longa, is a major component of turmeric and is commonly used as a spice and food-colouring agent by more than 1 billion people $[6,11]$. Curcumin has a surprisingly wide range of beneficial properties, including anti-inflammatory, antioxidant, chemopreventive and chemotherapeutic activity [4]. It exhibits a great promise as a therapeutic agent, and is currently used in human clinical trials for a variety of conditions, including heart diseases [18].

This study was performed to investigate whether oral curcumin treatment has any protective effect on cardiac myosin-induced autoimmune myocarditis in rats and to demonstrate the possible changes in TRX expression in cardiomyocytes following curcumin treatment.

\section{MATERIALS AND METHODS}

The study was conducted in the Department of Anatomy, College of Medicine, King Saud University, Riyadh, Kingdom of Saudi Arabia. The study was approved by the Research Ethical Committee of the College of Medicine, King Saud University (approval No. 11/2882/IRB).

\section{Animals}

Twenty-four six-week-old male Wistar rats, weighing about 200-250 g, were housed under standard environmental conditions with free access to standard pelleted rat chow and tap water. The study followed the International Guidelines for the Care and Use of Laboratory Animals for experimental procedure.

\section{Study design}

Animals were randomly allocated into 4 groups of 6 rats each.

- Group I (untreated control group) - rats received neither curcumin nor myosin;

- Group II (curcumin-exposed group) - rats received curcumin in water, orally, at the dose of $1 \mathrm{~g} / \mathrm{kg} /$ day [6], from day 1 to day 21;

- Group III (myocarditis group) - rats were immunised with $1 \mathrm{mg}$ of porcine cardiac myosin on day 1 and day $8[3,10,12,14]$;

- Group IV (myocarditis-curcumin group) - rats were immunised with $1 \mathrm{mg}$ of porcine cardiac myosin on day 1 and day 8 , and received curcumin in water, orally, at the dose of $1 \mathrm{~g} / \mathrm{kg} / \mathrm{day}$, from day 1 to day 21 .

All animals were sacrificed on day 22 by cervical decapitation under ether anaesthesia. Truncal blood was collected and serum was separated from each animal for biochemical assay. The hearts were excised and processed for histological and immunohistochemical studies.

Induction of EAM by immunisation. The rats were injected subcutaneously, in the back, on days 1 and 8 , with $1 \mathrm{mg}(0.1 \mathrm{~mL})$ of purified porcine cardiac myosin (10 mg/mL) (M 0531-10MG, Myosin, calcium activated, from porcine heart; buffered aqueous glycerol solution $1.05 \mathrm{~mL}$; $9.5 \mathrm{mg}$ protein/mL, Sigma-Aldrich Chemical Company, St. Louis, MO, USA) emulsified in an equal volume of complete Freund's adjuvant supplemented with mycobacterium tuberculosis H37Ra (DifcoLab., Detroit, MI, USA). Both control and control-curcumin groups were injected with complete Freund's adjuvant alone. 


\section{Histological study}

The excised hearts were fixed in 10\% buffered formalin at $4^{\circ} \mathrm{C}$ for $24 \mathrm{~h}$ and processed to prepare transverse mid-ventricular 5 - $\mu \mathrm{m}$-thick paraffin sections. These sections were stained with haematoxylin and eosin (H\&E) (Haematoxylin, Sigma, Life Science, product code 1001011134, USA — Eosin Y, Sigma-Aldrich, Batch \#01005 LE, USA). Myocarditis was determined by identifying both infiltrating mononuclear cells and necrosis of cardiomyocytes [8]. The percentage of myocarditis was assessed semiquantitatively, according to the scale: $0-$ normal; $1-$ mild $(<5 \%$ of heart cross-section involved); 2 - moderate (5-10\% of cross-section involved); 3 - marked (10-25\% of cross-section involved); and 4 - severe ( $>25 \%$ of cross-section involved) $[7,8]$.

\section{Immunohistochemical study}

Immunostaining of mid-ventricular paraffin sections of the heart for detection of TRX [14] was performed using streptavidin-biotinylated horseradish peroxidase (S-ABC) method (Novalink Max Polymer detection system, Novocastra, product NO. RE7280-K). The procedure involved the following steps: endogenous peroxidase activity was inhibited by $3 \% \mathrm{H}_{2} \mathrm{O}_{2}$ in distilled water for $5 \mathrm{~min}$, and then the sections were washed in Tris buffered saline (TRS) (Sigma, T 5030-100 TAB, pH 7.6) for $10 \mathrm{~min}$. Non-specific binding of antibodies was blocked by incubation with protein block for 5 min (Novocastra). Sections were incubated with rabbit polyclonal anti-rat TRX, Abcam, Cat\# ab26320 (100 $\mu \mathrm{g}, 0.4 \mathrm{mg} / \mathrm{mL})$, diluted 1:200 for $1 \mathrm{~h}$ at room temperature. Sections were washed in Tris buffer for 3 times, each for $3 \mathrm{~min}$, and then incubated with biotinylated anti-rabbit lgG (Novocastra) for $30 \mathrm{~min}$. This was followed by washing in Tris buffer for 3 times, each for $3 \mathrm{~min}$, and then incubated with Novolink polymer (Novocastra) for $30 \mathrm{~min}$. Then sections were washed in Tris buffer for 3 times, each for $3 \mathrm{~min}$. Peroxidase was detected with working solution of diaminobenzedine (DAB) substrate (Novocastra) for 10 $\mathrm{min}$. Finally, sections were washed in distilled water for $10 \mathrm{~min}$, nuclei were stained with Mayer's haematoxylin and sections were mounted in DPX (Dystyrene, Plasticizer, Xylene). For negative control sections, the same procedure was followed with omission of incubation in rabbit polyclonal anti-rat TRX.

\section{Image analysis}

High-resolution whole-slide digital scans of all TRX-immunostained sections were created with a ScanScope scanner (Aperio Technologies, Inc.).
The digital slide images were then viewed and analysed using the viewing and image analysis tools of Aperio's ImageScope software. Five areas, each with the fixed size of $137,000 \mu \mathrm{m}^{2}$, were randomly selected per section. To quantify the immunopositivity, the colour deconvolution (colour separation) algorithm (Aperio Technologies, Inc.) was set up (by colour calibration) so as to detect and quantify only the brown colour of DAB positive staining. The algorithm was then run on the selected area to measure the percentage of immumopositivity relative to the area being measured. The analysis output results were finally exported to Excel sheets and subjected to statistical analysis.

\section{Serum biochemical analysis}

Determination of cardiac injury markers levels. Serum troponin-T concentration was determined using a Siemens Dimension Xpand ${ }^{\circledR}$ Plus instrument (IL, USA). Serum creatine phosphokinase MB isoenzyme (CPK-MB) level was measured with an auto-analyser (ILab-300 bioMérieux Diagnostics, Milan, Italy).

Determination of tumour necrosis factor-alpha (TNF- $\alpha$ ) level. The concentration of the inflammatory cytokine TNF- $\alpha$ in serum was determined using commercially available enzyme-linked immunosorbent assay ELISA kits following the instructions supplied by the manufacturer (DuoSet kits, R\&D Systems; Minneapolis, MN, USA). The results were shown as pg of cytokine per $\mathrm{mL}$.

Determination of interleukin-6 (IL-6) level. The IL-6 levels were measured by ultra-sensitive ELISA (Quantikine HS Human IL-6 Immunoassay; R\&D Systems, Minneapolis, MN) with an analytical concentration value of $6.3 \%$ and a detection level of $0.04 \mathrm{pg} / \mathrm{mL}$ [5].

\section{Statistical analysis}

Data collected were subjected to statistical analysis using IBM SPSS Statistics version 22 software. The homogeneity of the obtained numerical data was first checked with Levene test and the homogeneity of variance assumption has been met. Analysis of variance (ANOVA) was used for an overall comparison between the study groups followed by Bonferroni as a post-hoc test for pairwise comparisons. Differences were considered significant when $p$ was equal to or less than 0.05 . A $95 \%$ confidence level was used to calculate a confidence interval, which is a range of values around the mean where the "true" (population) mean can be expected to be located, with $95 \%$ certainty. 


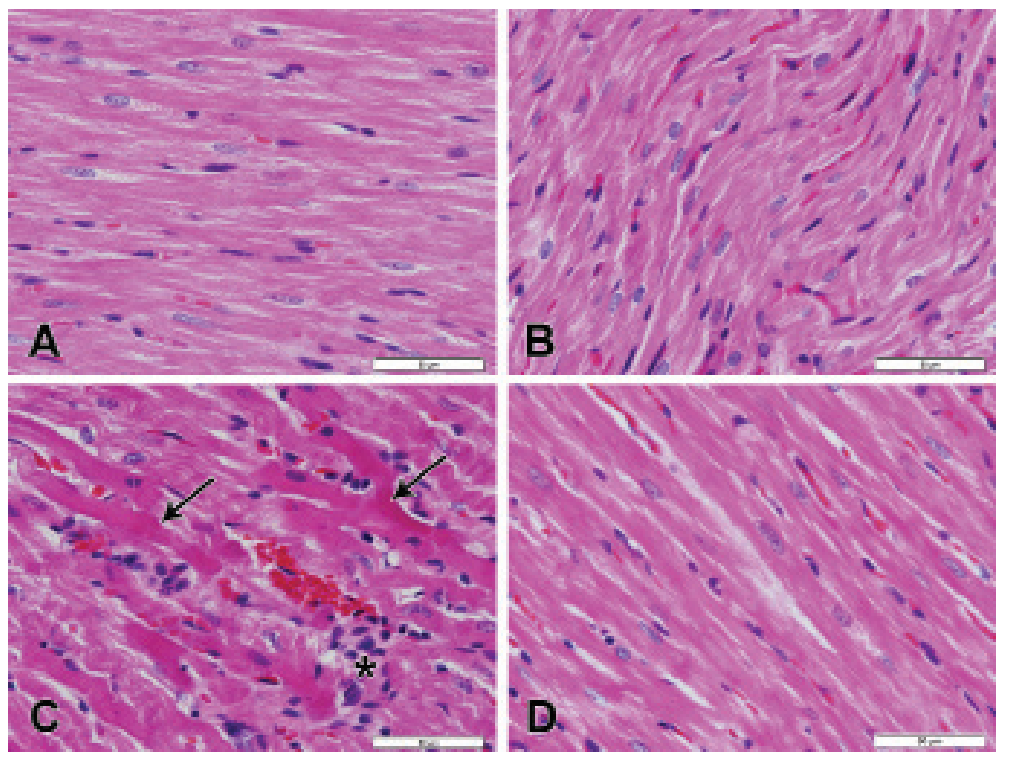

Figure 1. Heart sections stained with haematoxylin and eosin. A. Heart of rat from untreated control group; B. Heart of rat from curcumin-exposed group, showing normal architecture; C. Heart of rat from myocarditis group, showing necrotic cardiomyocytes (arrows); D. Heart of rat from myocarditis-curcumin group, showing normal architecture. Scale bars $=50 \mu \mathrm{m}$.

Table 1. Percentage of pyknotic nuclei in cardiomyocytes

\begin{tabular}{|c|c|c|c|c|c|c|c|c|}
\hline & & & $\begin{array}{r}\text { Gro } \\
\text { Control- }\end{array}$ & $\begin{array}{l}\text { p II: } \\
\text { urcumin }\end{array}$ & $\begin{array}{l}\text { Gros } \\
\text { Myoc }\end{array}$ & & $\begin{array}{r}\text { Gro } \\
\text { Myocardit }\end{array}$ & $\begin{array}{l}\text { V: } \\
\text { urcumin }\end{array}$ \\
\hline & Mean \pm SD & $95 \% \mathrm{Cl}$ & Mean \pm SD & $95 \% \mathrm{Cl}$ & Mean \pm SD & $95 \% \mathrm{Cl}$ & Mean \pm SD & $95 \% \mathrm{Cl}$ \\
\hline $\begin{array}{l}\text { Mean } \\
\pm \text { SD }\end{array}$ & $\begin{array}{c}0.000 \\
\pm 0.000\end{array}$ & $0.000-0.000$ & $\begin{array}{c}0.000 \\
\pm 0.000\end{array}$ & $0.000-0.000$ & $\begin{array}{r}58.468 \\
\pm 4.976\end{array}$ & $\begin{array}{l}53.246- \\
-63.690\end{array}$ & $\begin{array}{l}31.244 \\
\pm 5.670\end{array}$ & $\begin{array}{l}27.188- \\
-35.300\end{array}$ \\
\hline P1 & & & 1.000 & & $0.0002^{*}$ & & $0.0002^{*}$ & \\
\hline P2 & 1.000 & & & & $0.0002^{*}$ & & $0.0002^{*}$ & \\
\hline P3 & $0.0002^{*}$ & & $0.0002^{*}$ & & & & $0.0002^{*}$ & \\
\hline
\end{tabular}

*Significant difference $(\mathrm{p} \leq 0.05) ; \mathrm{Cl}$ — confidence interval; SD — standard deviation; P1 vs. Group I; P2 vs. Group II; P3 vs. Group III

\section{RESULTS}

The body weight of animals of myocarditis group was decreased, while the body weight of the animals of the other groups was markedly increased. The daily food and water consumption was reduced in animals of myocarditis group compared to the animals of the other groups. There was a marked decrease in the movements and activities of animals of myocarditis group compared to the animals of the other groups.

\section{Histological study}

H\&E-stained sections of the wall of the mid-ventricular level of the heart from all rats of Group I and Group II showed normal architecture of cardiomyocytes and endomysium (Fig. 1A, B). There were no pyknotic nuclei in the cardiomyocytes of both groups (Table 1). H\&E-stained sections of all rats of Group III showed evidence of severe myocarditis, in the form of inflammatory cell infiltration, swelling of cardiomyocytes, interstitial oedema, increased sarcoplasmic eosinophilia and increased number of cardiomyocytes with pyknotic nuclei (Fig. 1C). The mean percentage of pyknotic nuclei in cardiomyocytes of autoimmune myocarditis animals was $58.468 \pm 4.976 \%$ of the total number of cardiomyocytes (Table 1). H\&E-stained sections of rats of Group IV showed normal architecture in almost all cardiomyocytes and endomysium (Fig. 1D). Mild focal myocarditis ( $<5 \%$ of the total areas of the examined sections) was evident in the sections of this group. The mean percentage of pyknotic nuclei in cardiomyocytes showed a significant decrease to $31.244 \pm 5.670 \%$ compared to Group III (Table 1). 
Table 2. Thioredoxin expression by immunohistochemistry (area percent of strong positivity) in cardiomyocytes

\begin{tabular}{|c|c|c|c|c|c|c|c|c|}
\hline & \multicolumn{2}{|c|}{$\begin{array}{l}\text { Group I: } \\
\text { Control }\end{array}$} & \multicolumn{2}{|c|}{$\begin{array}{c}\text { Group II: } \\
\text { Control-curcumin }\end{array}$} & \multicolumn{2}{|c|}{$\begin{array}{l}\text { Group III: } \\
\text { Myocarditis }\end{array}$} & \multicolumn{2}{|c|}{$\begin{array}{c}\text { Group IV: } \\
\text { Myocarditis-curcumin }\end{array}$} \\
\hline & Mean \pm SD & $95 \% \mathrm{CI}$ & Mean \pm SD & $95 \% \mathrm{Cl}$ & Mean \pm SD & $95 \% \mathrm{Cl}$ & Mean \pm SD & $95 \% \mathrm{Cl}$ \\
\hline $\begin{array}{l}\text { Mean } \\
\pm \text { SD }\end{array}$ & $\begin{array}{c}16.003 \\
\pm 4.139\end{array}$ & $\begin{array}{l}12.543- \\
-19.463\end{array}$ & $\begin{array}{r}14.660 \\
\pm 9.977\end{array}$ & $\begin{array}{l}7.523- \\
-21.797\end{array}$ & $\begin{array}{l}39.777 \\
\pm 7.297\end{array}$ & $\begin{array}{l}32.119 \\
-47.435\end{array}$ & $\begin{array}{l}12.678 \\
\pm 3.808\end{array}$ & $\begin{array}{c}9.954- \\
-15.402\end{array}$ \\
\hline P1 & & & 0.976 & & $0.0002^{*}$ & & 0.738 & \\
\hline P2 & 0.976 & & & & $0.0002^{*}$ & & 0.916 & \\
\hline P3 & $0.0002^{*}$ & & $0.0002^{*}$ & & & & $0.0002^{*}$ & \\
\hline
\end{tabular}

${ }^{*}$ Significant difference $(\mathrm{p} \leq 0.05)$; $\mathrm{Cl}$ — confidence interval; SD — standard deviation; P1 vs. Group I; P2 vs. Group II; P3 vs. Group III
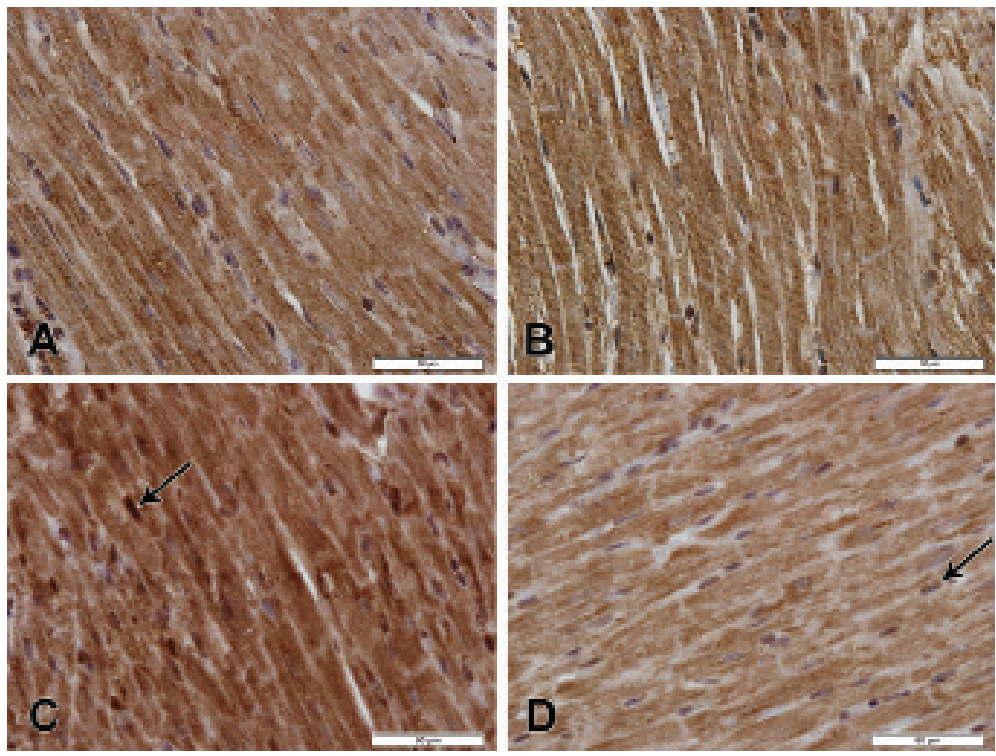

Figure 2. Heart sections immunostained with anti-thioredoxin antibody; A. Heart of rat from untreated control group; B. Heart of rat from curcumin-exposed group, all showing moderate immunoreactivity; C. Heart of rat from myocarditis group, showing intense immunoreactivity in sarcoplasm and nuclei (arrow); D. Heart of rat from myocarditis-curcumin group, showing moderate immunoreactivity of the sarcoplasm and few nuclei (arrow). Scale bars $=50 \mu \mathrm{m}$.

\section{Immunohistochemical study}

Immunohistochemistry was performed to determine the histological localisation of TRX in the heart. Sections of the heart from Group I and Group II showed moderate immunoreactivity for TRX in the sarcoplasm, while nuclei of cardiomyocytes were not immunostained. The extent of immunopositivity, expressed as mean area per cent of strong positivity, in Group I and Group II measured $16.003 \pm 4.139 \%$ and $14.660 \pm 9.977 \%$, respectively (Table 2). All sections of the heart from Group III showed strong immunoreactivity for TRX in the sarcoplasm and almost all nuclei of cardiomyocytes (Fig. 2C). This group showed significant increase in the mean area per cent of strong positivity to $39.777 \pm 7.297 \%$, compared to Group I and Group II (Table 2). Sections of the heart from
Group IV showed moderate immunoreactivity for TRX in the sarcoplasm of cardiomyocytes, with few positively immunostained nuclei of cardiomyocytes (Fig. 2D). The mean area per cent of strong positivity in this group was significantly decreased to $12.678 \pm 3.828 \%$ compared to Group III. There were no significant differences in the mean area per cent of strong positivity between this group and both untreated control and curcumin-exposed groups (Table 2).

\section{Biochemical study}

Serum cardiac injury markers, namely troponin-T and CPK-MB, were significantly increased in Group III as compared to Group I and Group II. The intake of curcumin in autoimmune myocarditis group sig- 
Table 3. Biochemical parameters in the studied groups

\begin{tabular}{lcccc}
\hline Groups & CPK-MB [U/L] & Troponin-T [pg/mL] & TNF- $\alpha$ [pg/mL] & Interleukin-6 [pg/mL] \\
\hline Group I (untreated control) & $33.71 \pm 3.89^{\mathrm{a}}$ & $7.35 \pm 1.53^{\mathrm{a}}$ & $6.69 \pm 1.13^{\mathrm{a}}$ & $9.32 \pm 0.44^{\mathrm{a}}$ \\
Group II (curcumin-exposed) & $37.79 \pm 5.28^{\mathrm{a}}$ & $6.95 \pm 0.87^{\mathrm{a}}$ & $6.34 \pm 0.59^{\mathrm{a}}$ & $8.27 \pm 1.25^{\mathrm{a}}$ \\
Group III (myocarditis) & $174.58 \pm 5.57^{\mathrm{b}}$ & $40.82 \pm 4.36^{\mathrm{b}}$ & $26.75 \pm 4.14^{\mathrm{b}}$ & $29.81 \pm 4.86^{\mathrm{b}}$ \\
Group IV (myocarditis-curcumin) & $131.13 \pm 2.37^{\mathrm{c}}$ & $30.87 \pm 1.94^{\mathrm{c}}$ & $16.69 \pm 1.57^{\mathrm{c}}$ & $18.21 \pm 1.57^{\mathrm{c}}$ \\
\hline
\end{tabular}

Each value represents the mean \pm standard deviation. The mean values not sharing a superscript alphabet in a column indicate significant difference at $\mathrm{p}<0.05$; CPK-MB - creatine phosphokinase MB isoenzyme; TNF- $\alpha$ - tumour necrosis factor alpha

nificantly down-modulated the deviation in these markers compared to myocarditis group (Table 3 ). However, the values were still significantly higher than those of control and control-curcumin groups. No significant differences were observed between Group I and Group II. Serum pro-inflammatory biomarkers (TNF- $\alpha$ and IL-6) were elevated significantly in sera of Group III as compared to Group I and Group II. The administration of curcumin to rats with myocarditis significantly reduced the inflammatory cytokines levels compared to myocarditis group. However, their levels were still significantly higher than those of Group I and Group II. No significant differences were observed between the Group I and Group II (Table 3).

\section{DISCUSSION}

Experimental autoimmune myocarditis in rat models is characterised by severe myocardial changes with inflammatory cell infiltration [12]. Excessive oxidative stress, caused by either increased ROS production or inadequate antioxidant defences, can lead to cardiovascular diseases [9]. Excessive oxidant stress leads to the release of inflammatory cytokines and chemokines that regulate leucocyte trafficking into the heart. Finally, ROS can destroy cells by necrosis or apoptosis, leading to further myocardial injury [15].

Curcumin is the active ingredient in the traditional herbal remedy and dietary spice turmeric (Curcuma longa). Curcumin has a wide range of beneficial properties, including inflammatory, antioxidant, chemopreventive and chemotherapeutic activity [4]. The pleiotropic activities of curcumin are derived from its complex chemistry, as well as, its ability to influence multiple signalling pathways. Curcumin is a free radical scavenger and hydrogen donor that exhibits both pro- and antioxidant activities. Furthermore, curcumin is remarkably non-toxic and exhibits limited bioavailability [6]. Studies performed on rats have confirmed a lack of significant toxicity of curcumin when admini- stered orally with doses up to $5 \mathrm{~g} / \mathrm{kg} /$ day [17]. To the best of the authors' knowledge, the dose selected in the present study is the lowest dose of curcumin used.

In the present study, immunohistochemistry for TRX was performed to examine the possible involvement of a redox regulating mechanism in the pathogenesis of immune-mediated myocarditis. Sections of the heart from myocarditis group showed a significant increase in the mean area per cent of strong positivity for TRX compared to control and control-curcumin groups. Shioji et al. [14] suggested that enhanced TRX expression may be induced by ROS produced by infiltrating inflammatory cells in acute immune-mediated myocarditis which, in turn, induce translocation of TRX from the cytoplasm into the nucleus. The authors reported that TRX might have a protective role against the progressive myocardial damage in acute immune-mediated myocarditis. Such protective role could be explained by the ability of TRX to decrease protein oxidation, chemokine signalling, and leucocyte trafficking [16]. One simple explanation for the beneficial effect of TRX is that it scavenges ROS that would oxidise proteins, lipids and DNA [1]. Sections of the heart from myocarditis-curcumin group showed that the mean area of TRX expression in this group was significantly decreased compared to myocarditis group, while no significant difference was shown between myocarditis-curcumin and both untreated control and curcumin-exposed groups. The results of the present study suggested that administration of curcumin ameliorates cardiac myosin-induced autoimmune myocarditis in rats, as evidenced by the decrease in TRX expression in that case. The present suggestion was also supported by the significant decrease in the serum levels of both cardiac injury markers (troponin-T and CPK-MB) and pro-inflammatory biomarkers (TNF- $\alpha$ and IL-6) shown in curcumin-myocarditis group compared with myocarditis group. The effect of curcumin could be attributed to its antioxidant and anti-inflammatory properties. However, the precise mechanism of the protective effect of curcumin in the acute phase of autoimmune myocarditis 
needs further investigation. It has been shown that curcumin significantly decreased plasma levels of IL-8, IL-10, TNF- $\alpha$ and cardiac troponin I in cases of rabbits underwent cardiopulmonary bypass (CPB). Furthermore, the appearance of apoptotic cardiomyocytes significantly decreased in the curcumin-CPB group. It was concluded that curcumin ameliorated the surge of pro-inflammatory cytokines during CPB and decreased the occurrence of cardiomyocytic apoptosis after cardiac injury [19]. Regarding the limitations of the present study, the authors studied only the effect of curcumin on acute myocarditis. Therefore, a future study on chronic phase of autoimmune myocarditis would be essential. Also, measurement of the serum levels of TRX would be more accurate than TRX immunoreactivity in the wall of the heart performed in the present study. Due to the low number of evaluated animals in each experimental group, the obtained data will be selected only as supporting data in the human risk evaluation.

\section{CONCLUSIONS}

In conclusion, the present study demonstrated that oral administration of curcumin ameliorates cardiac myosin-induced autoimmune myocarditis in rats, as evidenced by a significant reduction of TRX immunoreactivity, as well as, of the serum levels of both cardiac injury markers (troponin-T and CPK-MB) and pro-inflammatory biomarkers (TNF- $\alpha$ and IL-6). The authors would recommend the use of curcumin as a prophylactic and therapeutic agent for acute autoimmune myocarditis in human and encourage the estimation of serum level of TRX as a relevant indicator for the evaluation of the progress of acute myocarditis cases.

\section{ACKNOWLEDGEMENTS}

The authors gratefully acknowledge the Research Centre, College of Medicine, Deanship of Scientific Research, King Saud University, Riyadh, KSA for the financial support and continuous encouragement.

\section{REFERENCES}

1. Arner E, Holmgren A (2000) Physiological functions of thioredoxin and thioredoxin reductase. Eur J Biochem, 267: 6102-6109.

2. Blauwet L, Cooper L (2010) Myocarditis. Prog Cardiovasc Dis, 52: 274-288.

3. Futamatsu H, Suzuki J, Kosuge H, Yokosei O, Kamad M, Ito H (2003) Attenuation of experimental autoimmune myocarditis by blocking activated T cells through inducible costimulatory molecule pathway. Cardiovasc Res, 59: 95-104.
4. Hatcher H, Planalp R, Cho J, Torti FM (2008) Curcumin: from ancient medicine to current clinical trials. Cell Mol Life Sci, 65: 1631-1652.

5. Kaden J (2007) IL-6 determination in serum of kidney graft recipients by a new bedside test: its diagnostic relevance. Transplant Proc, 39: 511-513.

6. Kohli K, Ali J, Ansari M J, Raheman Z (2005) Curcumin: A natural anti-inflammatory agent. Indian J Pharacol, 37: 141-147.

7. Li Y, Heusr J, Kosanke S, Hemric M, Cunningham M W (2005): Protection against experimental autoimmune myocarditis is mediated by interleukin-10-producing $T$ cells that are controlled by dendritic cells. Am J Pathol, 167: 5-15.

8. Liu W, Nakamura H, Shioji K, Tanito M, Oka S, Ahsan MK, Son A, Ishii Y, Kishimoto C, Yodoi J (2004) Thioredoxin-1 ameliorates myosin-induced autoimmune myocarditis by suppressing chemokine expressions and leucocyte chemotaxis in mice. Circulation, 110: 1276-1283.

9. Lowenstein C J (2004) Exogenous thioredoxin reduces inflammation in autoimmune myocarditis. Circulation, 110: 1178-1179.

10. Matsui $Y$, Okamoto $H$, Jia N, Akino M, Uede T, Kitabatake A, Nishihira J (2004) Blockade of macrophage migration inhibitory factor ameliorates experimental autoimmune myocarditis. J Mol Cell Cardiol, 37: 557-566.

11. Menon VP, Sudheer AR (2007) Anti-oxidant and anti-inflammatory properties of curcumin. Advances in Experimental Medicine and Biology, 595: 105-125.

12. Otsuka K, Terasaki F, Ikemoto M, Fujita S, Tsukada B, Katashima T, Kanzaki Y, Sohmiya K, Kono T, Toko H, Fujita M, Kitaura $Y$ (2009) Suppression of inflammation in rat autoimmune myocarditis by S100A8/A9 through modulation of the pro-inflammatory cytokine network. Eur J Heart Fail, 11: 229-237.

13. Rosenstein E, Zucker M, Kramer N (2000) Giant cell myocarditis: most fatal of autoimmune disease. Semin Arthritis Rheum, 30: 1-16.

14. Shioji K, Kishimoto C, Nakamura H (2000) Up-regulation of thioredoxin (TRX) expression in giant cell myocarditis in rats. FEBS Lett, 472: 109-113.

15. Tao L Gao E, Coletti C, Wang Y, Christopher T, Lopez B, Koch W, Ma X (2006) Thioredoxin reduces post-ischemic myocardial apoptosis by reducing oxidative/nitrative stress. Br J Pharmacol, 149: 311-318.

16. Turoczi T, Chang VW, Engelman RM, Maulik N, Ho Y, Das DK (2003) Thioredoxin redox signaling in the ischemic heart: an insight with transgenic mice overexpressing Trx1. J Mol Cell Cardiol, 35: 695-704.

17. Wahlstrom B, Blennow G (1978) A study on the fate of curcumin in the rat. Acta Pharmacol Toxicol, 43: 86-92.

18. Wongcharoen W, Jai-Aue S, Phrommintikul A, Nawarawong W, Woragidpoonpol S, Tepsuwan T, Sukonthasarn A, Apaijai N, Chattipakorn N (2012) Effects of curcuminoids on frequency of acute myocardial infarction after coronary artery bypass grafting. Am J Cardiol, 110: 40-44.

19. Yeh $\mathrm{CH}$, Chen TP, Wu YC, Lin YN, Jing Lin P (2005) Inhibition of NF-kappaB activation with curcumin attenuates plasma inflammatory cytokines surge and cardiomyocytic apoptosis following cardiac ischemia/reperfusion. J Surg Res, 125: 109-116.

20. Yuan Z, Kishimoto C, Shioji K, Nakamura H, Yodoi J, Sasayama S (2003) Temocapril treatment ameliorates autoimmune myocarditis associated with enhanced cardiomyocyte thioredoxin expression. Mol Cell Biochem, 248: 185-192. 